\title{
Vowel Articulation Dynamic Stability Related to Parkinson's Disease Rating Features: Male Dataset
}

\author{
Pedro Gómez-Vilda* \\ Neuromorphic Speech Processing Lab, Center for Biomedical Technology \\ Universidad Politécnica de Madrid, Campus de Montegancedo \\ 28223 Pozuelo de Alarcón, Madrid, Spain \\ pedro@fi.upm.es \\ Zoltan Galaz and Jiri Mekyska \\ Department of Telecommunications, Brno University of Technology \\ Technicka 10, 61600 Brno, Czech Republic \\ José M. Ferrández Vicente \\ Universidad Politécnica de Cartagena, Campus Universitario Muralla del Mar \\ Pza. Hospital 1, 30202 Cartagena, Spain \\ Andrés Gómez-Rodellar \\ Neuromorphic Speech Processing Lab, Center for Biomedical Technology \\ Universidad Politécnica de Madrid, Campus de Montegancedo \\ 28223 Pozuelo de Alarcón, Madrid, Spain \\ Daniel Palacios-Alonso \\ Escuela Técnica Superior de Ingeniería Informática - Universidad Rey Juan Carlos \\ Campus de Móstoles, Tulipán, s/n, 28933 Móstoles, Madrid, Spain \\ Neuromorphic Speech Processing Lab, Center for Biomedical Technology \\ Universidad Politécnica de Madrid, Campus de Montegancedo \\ 28223 Pozuelo de Alarcón, Madrid, Spain \\ Zdenek Smekal \\ Department of Telecommunications, Brno University of Technology \\ Technicka 10, 61600 Brno, Czech Republic \\ Ilona Eliasova \\ First Department of Neurology, Faculty of Medicine \\ and St. Anne's University Hospital, Masaryk University \\ Pekarska 53, 65691 Brno, Czech Republic \\ Applied Neuroscience Research Group, Central European Institute of Technology \\ CEITEC, Masaryk University, Kamenice 753/5, 62500 Brno, Czech Republic \\ Milena Kostalova \\ Department of Neurology, Faculty Hospital and Masaryk University \\ Jihlavska 20, 63900 Brno, Czech Republic \\ Applied Neuroscience Research Group, Central European Institute of Technology \\ CEITEC, Masaryk University, Kamenice 753/5, 62500 Brno, Czech Republic
}

${ }^{*}$ Corresponding author.

This is an Open Access article published by World Scientific Publishing Company. It is distributed under the terms of the Creative Commons Attribution 4.0 (CC-BY) License. Further distribution of this work is permitted, provided the original work is properly cited. 


\author{
Irena Rektorova \\ First Department of Neurology, Faculty of Medicine and St. Anne's University Hospital \\ Masaryk University, Pekarska 53, 65691 Brno, Czech Republic \\ Applied Neuroscience Research Group, Central European Institute of Technology \\ CEITEC, Masaryk University, Kamenice 753/5, 62500 Brno, Czech Republic
}

Accepted 16 August 2018

Published Online 16 October 2018

\begin{abstract}
Neurodegenerative pathologies as Parkinson's Disease (PD) show important distortions in speech, affecting fluency, prosody, articulation and phonation. Classically, measurements based on articulation gestures altering formant positions, as the Vocal Space Area (VSA) or the Formant Centralization Ratio (FCR) have been proposed to measure speech distortion, but these markers are based mainly on static positions of sustained vowels. The present study introduces a measurement based on the mutual information distance among probability density functions of kinematic correlates derived from formant dynamics. An absolute kinematic velocity associated to the position of the jaw and tongue articulation gestures is estimated and modeled statistically. The distribution of this feature may differentiate PD patients from normative speakers during sustained vowel emission. The study is based on a limited database of 53 male PD patients, contrasted to a very selected and stable set of eight normative speakers. In this sense, distances based on Kullback-Leibler divergence seem to be sensitive to PD articulation instability. Correlation studies show statistically relevant relationship between information contents based on articulation instability to certain motor and nonmotor clinical scores, such as freezing of gait, or sleep disorders. Remarkably, one of the statistically relevant correlations point out to the time interval passed since the first diagnostic. These results stress the need of defining scoring scales specifically designed for speech disability estimation and monitoring methodologies in degenerative diseases of neuromotor origin.
\end{abstract}

Keywords: Neurodegenerative disorder; Parkinson's Disease; speech neuromotor activity; aging voice; hypokinetic dysarthria.

\section{Introduction}

Parkinson's Disease (PD) is an incurable, chronic, neurodegenerative disorder that is characterized by the neuropathological motor disorders resulting from alterations in sensory-motor circuitry consequent to neurotransmitter decay and imbalance, and pathological electrophysiological activities in basal ganglia, specifically in substancia nigra pars compacta $\frac{112}{12}$ PD has a major impact on patients' mobility, wellbeing, social life, communication, and integration in the society. In early stages, PD produces the primary motor symptoms such as tremor at rest, muscular rigidity, and progressive bradykinesia, $\frac{3}{3}$ followed by the secondary motor symptoms such as dysarthria, dysphagia, freezing of gait, etc ${ }^{4}$ In later stages, many patients eventually develop various nonmotor symptoms that are manifested in the behavioral and cognitive domains, such as conduct alterations, memory-related problems, anxiety, depression, emotional and cognitive impairments, etc.517] At present, PD is diagnosed in approximately $1.5 \%$ of people aged over 65 years, where age is the most significant onset factor. ${ }^{8}$ According to previous studies, ${ }^{9}$ up to $90 \%$ of patients diagnosed with PD exhibit the distinctive motor speech disorder characterized by poor respiratory function, rigidity, bradykinesia, and reduced muscular control of the larynx, articulation organs, and other physiological support mechanisms for speech, which is generally referred to as hypokinetic dysarthria (HD). Up to this point, increased acoustic tremor, rough and asthenic phonation, increased velo-pharyngeal nasality, monopitch, monoloudness, speech rate and fluency disturbances, reduced mobility of the articulation organs, involuntary introduction of pauses, rapid repetitions of words and syllables, and sudden deceleration or acceleration in speech have been observed in patients with PD. The reader can check Brabenec et al 10 for a comprehensive review. Classically, respiratory and phonation impairments associated with HD in PD have been exhaustively studied using a variety of conventional acoustic measures such as jitter, shimmer, ratios of harmonic and noise components in speech, glottal source features, etc., extracted from 
the sustained phonation of a vowel!10!11 It may be observed that few studies have been carried out with respect to the articulation impairment associated with HD in PD. Researchers have mainly focused on the analysis of impaired consonant articulation in diadochokinetic tasks employing acoustic features such as Voice Onset Time (VOT), or they monitored configuration of articulatory organs based on formant triangles (resonances of the oronaso-pharyngeal tract) and other formant-derived static features such as Vowel Space Area (VSA), Formant Centralization Ratio (FCR) or Vowel Articulation Index (VAI) ${ }^{[12]}$ In recent years, researchers have also tried to develop nonconventional acoustic measures that would be capable of capturing a complex voice/speech pathology which is present in the more advanced stages of the disease, when voice becomes noisy, irregular, chaotic and therefore hardly described by conventional acoustic measures. Nowadays, it is well-documented that nonconventional acoustic measures provide more precise HD identification. Nevertheless, these measures are in general less clinically interpretable. To assess and monitor PD progress, clinicians use a variety of rating scales, such as the Unified Parkinson's Disease Rating Scale (UPDRS) ${ }^{13}$ or the Hoehn \& Yahr scale ${ }^{14}$ However, these rating scales have not been specifically designed to take HD into account. On the one hand, to study the influence of disease progress, neurologists have resourced to other indices, as the Freezing of Gait Questionnaire (FOGQ) $\frac{15}{15}$ the Non-Motor Symptoms Scale (NMSS), 16 the REM sleep Behavioral Disorder Screening Questionnaire (RBDSQ) $\frac{1718}{1}$ the Beck Depression Inventory (BDI) $\frac{19}{19}$ the Mini Mental State Examination (MMSE) $\stackrel{20}{20}$ or the revised Addenbrooke's Cognitive Examination (ACE-R $)^{21}$ to evaluate the state of the patient under different points of view. On the other hand, having into account that PD is an illness characterized by the failure of the peripheral neuromotor activity, it could be possible that a description of the neuromotor activity, supported by features estimated from speech, could serve as a possible semantic descriptor of patient's conditions. A possible description of the neuromotor activity from speech can be given in terms of the dynamic changes experimented by the resonant frequencies of the vocal tract, which are known classically as formants. The aim of the present study is to evaluate if features derived from the dynamic behavior of formants in sustained vowels are related with some of these indices, and to establish to which extent dynamic measures can be used in the multimodal study of PD speech production. Initially, dynamic measurements on formant activity, as the absolute kinematic velocity (AKV), which will be defined in the sequel, seeming to be highly correlated with the superficial myoelectric activity of certain facial muscles ${ }^{22}$ may be the adequate candidates for such study. The structure of the present paper is as follows. Section 2 describes the cohort of patients, the biomechanical foundations explaining distortion of vowel articulation by means of formant dynamics, the information theory fundamentals behind the distance measurements used in distinguishing healthy and control utterances, and the methodology of consequent statistical processing. The results from the present work are shown in Sec. 3 and discussed in Sec. 4 Conclusions are given in Sec. 5 .

\section{Patients and Methods}

\subsection{Participants in the study}

The pathological database used is a part of the Parkinsonian Speech Database (PARCZ) recorded at St. Anne's University Hospital in Brno, Czech Republic, and consisted of four sets of five Czech vowels $(/ \mathrm{a}, \mathrm{e}, \mathrm{i}, \mathrm{o}, \mathrm{u} /)^{)}$pronounced in four different ways: short vowels and sustained vowels, both uttered in a natural way; sustained vowels uttered with maximum loudness, and with minimum loudness. The subset selected for the experiments described in the present paper corresponded to utterances of vowel [a:] at maximum loudness by 53 male PD patients (mean age $66.2 \pm 8.8$ years). For clinical data see Table 1. None of the patients had a disease affecting the central nervous system other than PD. All patients were examined on their regular dopaminergic medication (ON stage) approximately $1 \mathrm{~h}$ after the L-dopa dose. The study was approved by the local ethics committee, and all patients signed an informed consent form. The normative database consisted in utterances from vowel [a:] at normal level, from a set of 50 normative male subjects (mean age $30.83 \pm 10.37$ years) free from organic or neurologic pathology selected after inspection by the ENT services of Hospital Gregorio Marañón of Madrid. Recordings from eight of these subjects were used in the experiments as commented in the sequel. The 
Table 1. Patients' Clinical Data: UPDRS III Unified PD Rating Scale, part III: Motor Examination (Movement Disorder Society Task Force on Rating Scales for PD 2003), LED - L-dopa equivalent daily dose (mg/day), 24 NMSS - Non-Motor Symptoms Scale, 16 RBDSQ - REM sleep Behavioral Disorder Screening Questionnaire, 1718 MMSE Mini Mental State Examination, 20 ACE-R - Addenbrooke's Cognitive Examination revised, 21$]$ BDI - Beck Depression Inventory 19 FOG-Q — Freezing of Gait Questionnaire! 15

\begin{tabular}{lrrccc}
\hline \multicolumn{6}{c}{ Males } \\
Clinical data & Mean & Std & Min & Median & Max \\
\hline PD duration (yrs) & 7.9 & 4.3 & 1 & 7 & 21 \\
UPDRS III & 26.9 & 10.7 & 5 & 27 & 55 \\
LED (mg) & 1089.8 & 555.2 & 55.6 & 997.5 & 2275 \\
NMSS & 38.3 & 20.3 & 2 & 37 & 79 \\
RBDSQ & 3.9 & 3.1 & 0 & 3 & 12 \\
MMSE & 28.3 & 1.5 & 21 & 29 & 30 \\
ACE-R & 87.3 & 8.0 & 60 & 87 & 100 \\
BDI & 9.7 & 5.8 & 0 & 9 & 26 \\
FOG-Q & 6.9 & 5.9 & 0 & 7 & 20 \\
\hline
\end{tabular}

normative samples were selected using Mutual Information criteria to maximize variance and minimize redundancy.

The recordings were undersampled to $8 \mathrm{kHz}$, and the first two formants $F_{1}$ and $F_{2}$ were estimated by a combined technique detecting the maxima in the vocal tract transfer function $H(\omega)$ as by (1), and the zeros of the transfer function $B(z)$ in the complex plane as by

$$
\begin{array}{r}
\left\{F_{1}, F_{2}\right\}=\min _{2 \text { first }}\left\{\max _{\operatorname{local} \text { in } \omega}|H(\omega)|\right\} ; \\
H(\omega)=1 /\left[1-\sum_{i=1}^{k} b_{i} e^{-j i \omega \tau}\right],
\end{array}
$$

where $b_{i}$ are the coefficients of a $k$-order adaptive linear predictor, $\frac{23}{\omega} \omega$ is the angular frequency and $\tau$ is the sampling interval.

\subsection{Biomechanics of formant kinematics}

Speech production is planned and instantiated in the linguistic neuromotor cortex.25] The activity of cortical neurons (primary) is encoded as neuromotor actions in the basal ganglia, where secondary neurons connected to the muscles of the pharynx, tongue, larynx, chest and diaphragm through sub-thalamic secondary pathways produce sequences of motor actions which activate the respiratory, phonatory and articulatory systems responsible for speech production. Regarding articulation, the principal structures to consider are the jaw, tongue and lip muscles. In this work, the Jaw-Tongue Biomechanical System (JTBS), in Fig. 1 will be studied.

The dynamics of the JTBS $S^{26130131}$ can be approximated by a third-order lever fixed at the skull in $(F)$, allowing movements mainly in the sagittal plane $(x, y)$. For the purposes of articulation, it can be considered in a first approach as a joint lumped mass system subject to different forces actuating on the Jaw-Tongue Reference Point $P_{r \mathrm{JT}}\left(x_{r}, y_{r}\right)$. The main forces considered are the masseter up-lift $\left(f_{m}\right)$, the styloglossus pull-up-back $\left(f_{\mathrm{sg}}\right)$, the genio-hyoglossus pull-down-back $\left(f_{\mathrm{gh}}\right)$ and the gravity $\left(f_{w}\right)$. Besides, due to the action of genioglossus and glosso-intrinsic muscles $\left(f_{\mathrm{gi}}\right)$, the tongue blade and appex may be projected forwards. As a result, $P_{r \mathrm{JT}}$ will experience changes in both directions $\left(\Delta x_{r}, \Delta y_{r}\right)$. Associating jaw-tongue gestures with formants is not a simple

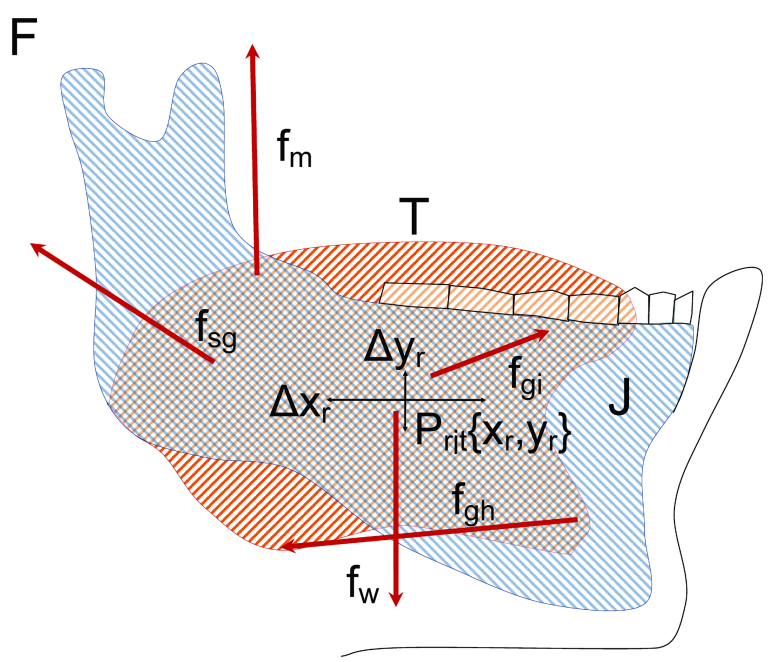

Fig. 1. Jaw-Tongue Biomechanical System. A reference point is defined at $P_{r \mathrm{JT}}$, where forces acting on the system (dynamics) induce movements in the sagittal plane (kinematics). Coordinates system: $x$ is rostral-caudal, and $y$ is dorsal-ventral. T: tongue; J: jaw; F: fulcrum; $f_{m}, f_{\mathrm{sg}}, f_{\mathrm{gi}}, f_{\mathrm{gh}}$ and $f_{w}$ refer to forces exerted by the masseter, styloglossus, intrinsic glossus, hyoglossus and gravity, respectively, whereas $\Delta x$ and $\Delta y$ refer to small displacements around the reference point in the rostralcaudal and dorsal-ventral directions. 
task, as the system acoustic properties are rather complex ${ }^{28}$ Nevertheless, a first-approach relationship could be as

$$
\begin{aligned}
{\left[\begin{array}{l}
\Delta F_{1}(t) \\
\Delta F_{2}(t)
\end{array}\right] } & =\mathbf{A}\left[\begin{array}{l}
\Delta x_{r}(t) \\
\Delta y_{r}(t)
\end{array}\right], \\
\mathbf{A} & =\left[\begin{array}{ll}
a_{11} & a_{12} \\
a_{21} & a_{22}
\end{array}\right],
\end{aligned}
$$

where $a_{i j}$ are the transformation weights associating $P_{r \mathrm{JT}}$ to formants, and $t$ is the time. The functional A expressing the relationship is known to be nonlinear, time-variant and multi-valued, i.e. the relation between $P_{r \mathrm{JT}}$ and formant values do not follow a linear rule (superposition could not be applied), the relationship would be time-dependent, $\mathbf{A}=$ $\mathbf{A}(t)$, and different articulation positions may produce identical formant pairs. Therefore, to facilitate a first-order approach study, the following assumptions had to be taken into account:

- A linear functional A could be considered provided that movement amplitude ranges are not large (small-signal approach). This hypothesis is based on the linearization of such functional in terms of first-order Taylor's expansions around a stable phonation position as $P_{r \mathrm{JT}}$, where matrix A could be represented by the Jacobian between formants and positions. ${ }^{300}$ It is well known that the Jacobian matrix of a function $\boldsymbol{f}$ defines the best linear approximation of the function $f$ near a reference point $\left(P_{r \mathrm{JT}}\right.$ in the present case).

- Time invariance could be granted if only lowfrequency movements are considered (i.e. if low frequency contents of dynamic variables are much larger in amplitude than high frequency ones), this compromise is granted because dynamic variables as $\Delta x_{r}$ and $\Delta y_{r}$ are harmonic oscillations around $P_{r \mathrm{JT}}$, and they are estimated by integration of accelerations and velocities, and if the estimation windows are short compared with low frequency contents (quasi-stationary approach). .3132

- The one-to-many association of formant positions could be handled provided that the joint probability between formant pairs and articulatory positions is carefully modeled for the utterances of interest. 29

- The functional $\mathbf{A}$ is invertible, i.e. that an inverse matrix exists: $\mathbf{W}=\mathbf{A}^{-1}$. This condition has been assessed for diadochokinetic oscillatory exercises 28 of about $1 \mathrm{~cm}$ wide around $P_{r \mathrm{JT}}$, and can be extended to low fluctuations produced by jaw tremor and related phenomena, as the ones found in the present set of PD subjects. These alterations show oscillation frequencies between $2-12 \mathrm{~Hz}$, for which high frequency amplitudes are much lower than the first harmonic.33

Under the above mentioned conditions, it will be possible to obtain average estimates of $a_{i j}$ for short time windows using linear regression between formant and displacement intervals by iterative approximations. Estimations of $a_{11}$ and $a_{12}$ may be obtained from

$$
\Delta F_{1}(t)=a_{11} \Delta x_{r}(t)+a_{12} \Delta y_{r}(t)
$$

by the following recursion:

$$
\begin{aligned}
& a_{11}^{k}=\frac{E\left\{\Delta F_{1}, \Delta F_{1}\right\}-\gamma c_{22}^{k-1} E\left\{\Delta F_{1}, \Delta x_{y}\right\}}{E\left\{\Delta F_{1}, \Delta x_{r}\right\}}, \\
& a_{12}^{k}=\frac{E\left\{\Delta F_{1}, \Delta F_{1}\right\}-\gamma c_{21}^{k-1} E\left\{\Delta F_{1}, \Delta x_{r}\right\}}{E\left\{\Delta F_{1}, \Delta x_{y}\right\}},
\end{aligned}
$$

where $0<\gamma<1$ is a convergence parameter, $k$ is the iteration index and $E\{f, g\}$ is the expectation between functions $f$ and $g$, subject to the following initial conditions:

$$
\begin{aligned}
& a_{11}^{0}=\frac{E\left\{\Delta F_{1}, \Delta F_{1}\right\}}{E\left\{\Delta F_{1}, \Delta x_{r}\right\}}, \\
& a_{12}^{0}=\frac{E\left\{\Delta F_{1}, \Delta F_{1}\right\}}{E\left\{\Delta F_{1}, \Delta x_{y}\right\}} .
\end{aligned}
$$

A relationship similar to (3) may be established for $\Delta F_{2}$

$$
\Delta F_{2}(t)=a_{21} \Delta x_{r}(t)+a_{22} \Delta y_{r}(t) .
$$

In general, the oscillations of both formants will be influenced by the fluctuations of $P_{r \mathrm{JT}}$.

On these premises, it will be possible to define an AKV of the reference point $P_{r \mathrm{JT}}$ associated to the first and second formant drifts.

$$
\begin{aligned}
\left|v_{r}(t)\right|= & {\left[B_{1}\left(\frac{\partial F_{1}}{\partial t}\right)^{2}+B_{2}\left(\frac{\partial F_{2}}{\partial t}\right)^{2}\right.} \\
& \left.+B_{12} \frac{\partial F_{1}}{\partial t} \frac{\partial F_{2}}{\partial t}\right]^{\frac{1}{2}},
\end{aligned}
$$

where $B_{1}, B_{2}$ and $B_{12}$ are quadratic forms of $\mathbf{W}$. 


\subsection{Mutual information divergence}

The AKV of the reference point given in (7) is a very semantic correlate, as it can be associated to streams of neuromotor actions involving phonation $\stackrel{33}{\text { It has }}$ been shown that its histogram-derived probability density function $p\left(v_{r}\right)$ contains information related to phonated intervals and pauses, syllable nuclei, vowel onsets and trails, and other dynamic features present in speech articulation. ${ }^{34}$ In Fig. 22 the AKV pdf's from a PD patient contrasted against the same distribution from a healthy control are shown. The control sample is the one with lowest distance to the model set (best case). The PD file is the one with the largest distance to the model set (worst case).

It may be seen that the PD distribution is spread over the whole speed span, whereas the distribution of the healthy control is limited under $20 \mathrm{~cm} \cdot \mathrm{s}^{-1}$, confirming the differential behavior of both types of distributions. Their Mutual Information contents can be estimated modifying Kullback-Leibler's Divergence $e^{35}$

$$
\begin{aligned}
D_{\mathrm{KL} i j} & \left\{p_{T i}\left(v_{r}\right), p_{M}\left(v_{r}\right)\right\} \\
= & \int_{\zeta=0}^{\infty} p_{M}(\zeta) \operatorname{abs}\left\{\log \left[\frac{p_{T i}(\zeta)}{p_{M}(\zeta)}\right]\right\} d \zeta,
\end{aligned}
$$

where $p_{\operatorname{Ti}}\left(v_{r}\right)$ is the pdf's of the $i$ th target subjects (patients), respective to the geometric mean of the $m=8$ model pdf's $p_{M}\left(v_{r}\right)$ as

$$
p_{M}\left(v_{r}\right)=\left[\prod_{j=1}^{m} p_{M j}\left(v_{r}\right)\right]^{\frac{1}{2}} ; \quad 1 \leq j \leq m,
$$

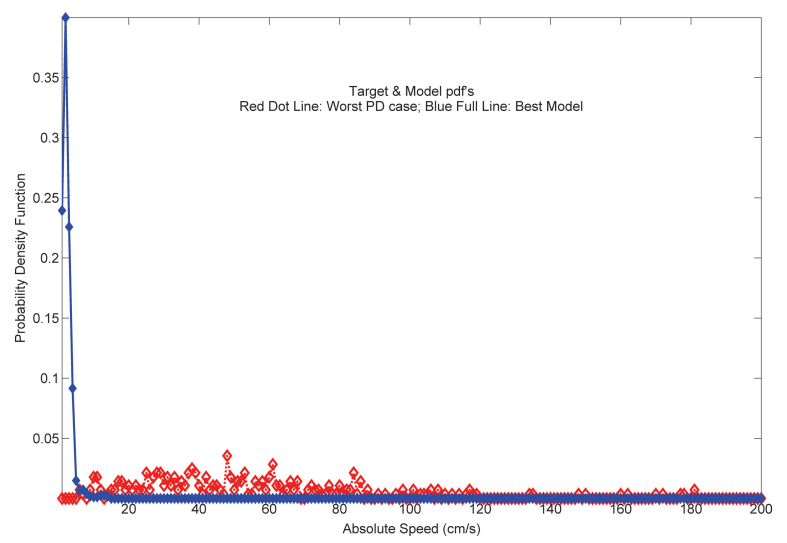

Fig. 2. (Color online) Probability density functions of the absolute kinematic velocity $v_{J}$ from a male healthy control (blue full line and circles) and a PD patient (red dot line and diamonds). The AKV is given in $\mathrm{cm} \cdot \mathrm{s}^{-1}$. where $\left\{M_{j}\right\}$ refers to the set of normative male subjects mentioned before, and the AKV defined in (7) is limited to positive real values $\left(v_{r} \in \mathbf{R}_{\geq \mathbf{0}}\right)$. In what follows, a study on PD vowel formant stability will be conducted to compare the results from the male population with the healthy controls.

\subsection{Statistical analysis}

The present study has a marked exploratory nature, as to our knowledge, vowel formant kinematics has not been used before in PD detection, grading or monitoring. The intention of the study is to show the performance of this methodology in population grading studies of PD patients. The algorithmic procedures are being described in the next steps:

- Fragments of $500 \mathrm{~ms}$ (estimation window) of sustained vowel [a:] were selected from the recordings under analysis, and downsampled to $8 \mathrm{kHz}$.

- $F_{1}$ and $F_{2}$ were estimated as by (1) using an adaptive linear predictor each $2 \mathrm{~ms}$. The frequency resolution was $2 \mathrm{~Hz}$.

- The AKV $\left(\left|v_{r}(t)\right|\right)$ was estimated taking (77) into account.

- An $N$-bin histogram of counts by amplitudes was built from each sample AKV. The speed interval was $\left[0,\left|v_{r}\right|_{\max }\right]$, with $\left|v_{r}\right|_{\max }=200 \mathrm{~cm} \cdot \mathrm{s}^{-1}$, therefore each bin was $\Delta b_{k}=\left[\left|v_{r}\right|_{\max } / N\right]=1 \mathrm{~cm} \cdot \mathrm{s}^{-1}$ wide.

- The following histogram count was built for each bin $b_{k}=k \cdot \Delta b_{k}$ :

$$
\text { if } b_{k-1} \leq v_{r}(t)<b_{k} \text { then } c_{k}=c_{k}+1
$$

$c_{k}$ being the number of counts for bin $b_{k}$.

- Count histograms $c_{k}(0 \leq k \leq N)$ were normalized to their total number of counts $C_{t}=\Sigma b_{k}$ (for all $b_{k}$ ), therefore, they could be considered estimators of probability density functions $p_{k}=c_{k} / C_{t}$.

- Kullback-Leibler Divergences $D_{\mathrm{KL}}$ were estimated following (8) using the normalized count histograms as it is described in the sequel.

The target and model $D_{\mathrm{KL}}$ with respect to eight normative male speakers are defined as

$$
\begin{gathered}
D_{\mathrm{KL}}\left(T_{i}, M\right)=D_{\mathrm{KL}}\left\{p_{T i}\left(v_{r}\right), p_{M}\left(v_{r}\right)\right\}, \\
D_{\mathrm{KL}}\left(M_{j}, M\right)=D_{\mathrm{KL}}\left\{p_{M j}\left(v_{r}\right), p_{M}\left(v_{r}\right)\right\},
\end{gathered}
$$

where $\{M\}$ refers to the sets of normative male subjects $M_{j}$ mentioned before. 
A subset of eight male subjects were selected on the condition of showing the largest accumulated $D_{\mathrm{KL} j}\left(M_{j}, M\right)$ to become the normative model set (see the explanation in Sec. 4). This model set was used to estimate the accumulated $D_{\mathrm{KL}}$ of $\mathrm{PD}$ patients against all the pdf's in the model set, as $(p=53, m=8)$

$$
\begin{array}{r}
D_{\mathrm{KL} i j}\left(T_{i}, M_{j}\right)=D_{\mathrm{KL}}\left\{p_{T i}\left(v_{r}\right), p_{M j}\left(v_{r}\right)\right\} ; \\
i \in[1, p] ; j \in[1, m] .
\end{array}
$$

The eight normative subjects were selected on the condition of showing the lowest $D_{\mathrm{KL}}\left(M_{j}, M\right)$. The probability distributions of eight normative male subjects and $53 \mathrm{PD}$ patients are shown in Fig. 3 It may be seen that healthy controls show activity under $20 \mathrm{~cm} \cdot \mathrm{s}^{-1}$, whereas PD patient distributions show activity spread over higher velocities, most of them confined under $100 \mathrm{~cm} \cdot \mathrm{s}^{-1}$, some of them reaching $200 \mathrm{~cm} \cdot \mathrm{s}^{-1}$. It seems evident that the extent of the probability density function is a clear hallmark to the presence of pathology. Normative speakers should show a much higher stability in formant production than PD patients. As probability density functions must enclose an area equal to the unity by definition, normative profiles must be confined to low speed limits, whereas PD profiles are supposed to

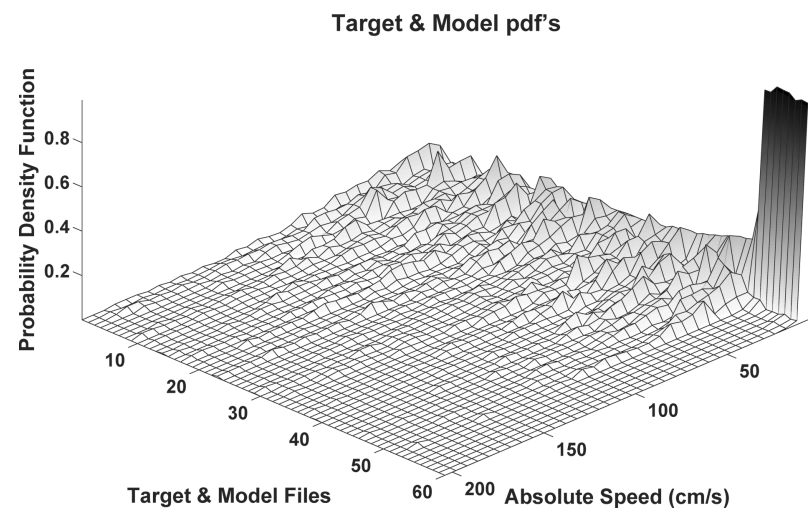

Fig. 3. Probability density functions of the AKV $p\left(v_{r}\right)$ from $53 \mathrm{PD}$ patients (files 1-53) and eight male healthy controls (files $1-8$ ). The AKV is given in $\mathrm{cm} \cdot \mathrm{s}^{-1}$. Normative distributions concentrate on the vertical axis, whereas PD distributions spread over the horizontal axis. The area under the curve in each distribution is the unity. This is a very relevant fact, as $D_{\mathrm{KL}}$ is defined in (8) it is based on estimating the overlap between some distributions concentrating near the origin (normatives) with others spread over a large part of the horizontal axis (pathological).

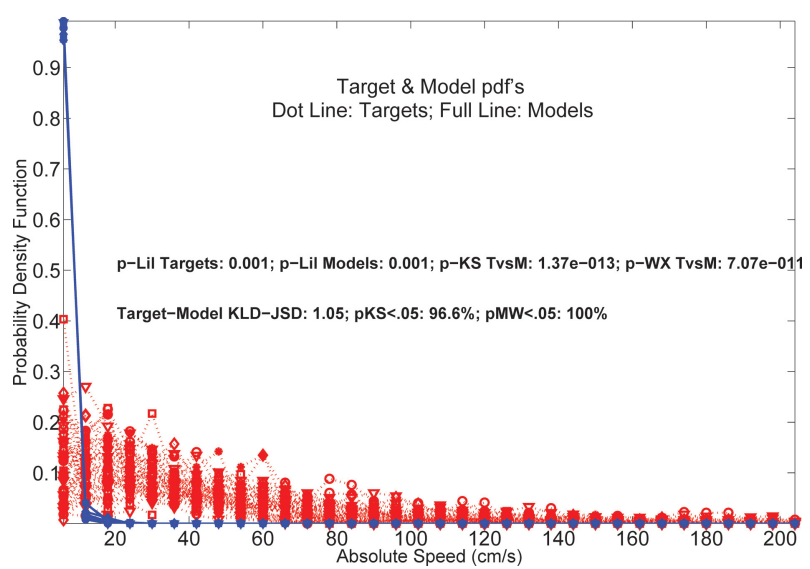

Fig. 4. (Color online) Superimposed probability density functions of the AKV $p\left(v_{r}\right)$ from 53 PD patients (files 153 ) and eight male healthy controls (files $1-8$ ). The AKV is given in $\mathrm{cm} \cdot \mathrm{s}^{-1}$. Results of different statistical tests are given in the figure (see text). The small dispersion of normative distributions (in blue) is contrasted against the large dispersion shown by pathological distributions (in red). The results of different tests to assess the nonGaussian character of these distributions, which show the behavior of $\chi^{2}$ distributions are given superimposed.

show lower values at the origin and be more spread over larger velocities. This situation is well reflected in Fig. 4, which shows the profiles of normative distributions in full line (blue circles) whereas the PD distributions are given in dash line (red diamonds). The difference in the profiles is evident. On its turn, Fig. 4 shows also the results of some tests passed on the probability density functions. For instance, the nonGaussian nature of the PD and normative distributions (targets and models, respectively) is confirmed by the rejection of the null hypothesis (H0: distributions being Gaussian) using Lilliefors tests (p-Lil Targets and Models under 0.05). The average target and model distributions have been tested for similarity (H0: similar distributions), confirming the rejection of the null hypothesis both by KolmogorovSmirnov and Wilcoxon tests ( $p$-Ks and $p$-WX for TvsM under 0.05).

The $D_{\mathrm{KL}}$ between targets and models is estimated by a Kolmogorov-Smirnov test (LKD-JSD) as 1.05. The percentage of target probability distributions rejecting the null hypothesis of similarity with respect to the model distributions under a $p$-value $<0.05$ is of $96.6 \%$ and $100 \%$ according to Kolmogorov-Smirnov and Mann-Whitney tests. 


\section{Results}

An important issue in monitoring pathology is that of grading, as short-term timely monitoring of PD may be highly relevant for patient treatment and rehabilitation. ${ }^{36}$ One of the intentions of the study was to relate $D_{\mathrm{KL}}$ (objective grading) with different clinic evaluation scales currently in use (subjective grading). At this point, to assess the relationship strength between the articulation instability measure and other motor/nonmotor PD symptoms Pearson's (linear relation) and Spearman's (monotonic relation) partial correlation coefficients (controlling for the effect of other clinical factors) between the $D_{\mathrm{KL}}$ and the scores of selected clinical scales were computed, within the significance level of correlation set to $p=0.05$. This same correlation analysis was carried out between $D_{\mathrm{KL}}$ and PD duration as well. The controlling factors for particular data are listed below:

- PD duration: age

- UPDRS III: age, LED, BDI

- FOG-Q: age, LED

- NMSS: age, LED, UPDRS III

- RBDSQ: age

- MMSE: age, LED, BDI, UPDRS III, education extent

- ACE-R: age, LED, BDI, UPDRS III, education extent

- BDI: age, LED, UPDRS III

Figure 5 shows the matrix of divergence $D_{\mathrm{KL}}$ from (10) between each patient in the target set $\left\{T_{i}\right\}$ with respect to each one in the model set $\left\{M_{j}\right\}$. The target and model sets have been ordered accordingly to the $D_{\mathrm{KL}}$ of the corresponding pdf's to the average model pdf $\{M\}$ as given in (11). Therefore, the first target file is supposed to be the less divergent with respect to the model set, whereas the 53rd target file should be the most divergent. In this way, an ordered set of files by divergence to the model set is produced. It may be seen also that the model files show a quite uniform behavior, as the distance of any target file to each one of them is very similar. The question now is finding out to which extent $D_{\mathrm{KL}}$ is related to subjective evaluation scales. The results of the partial correlations are given in Table 2

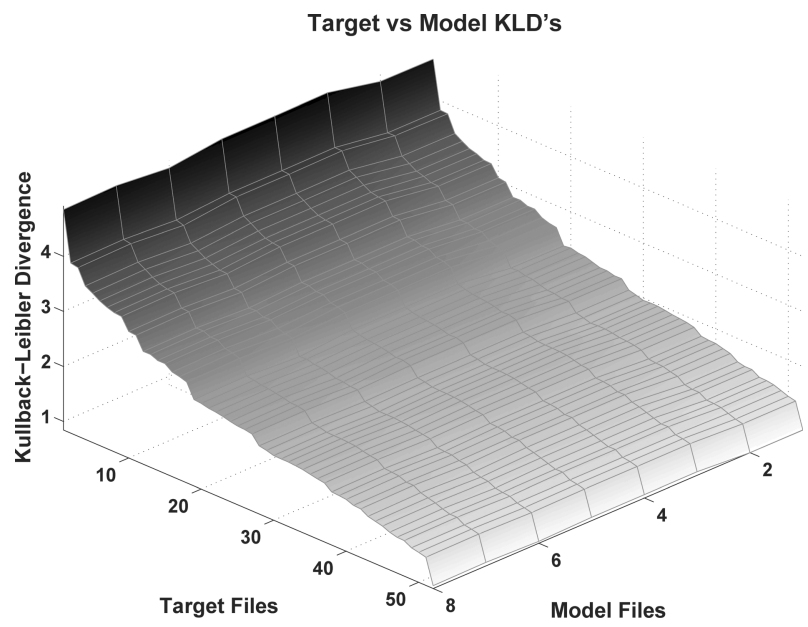

Fig. 5. Modified KL Divergence between 53 male PD patients (targets) and eight male healthy controls (models). The similarity of the divergence from a given target file to the eight healthy controls is a proof of the uniform characteristics of the controls.

Table 2. Partial correlations between DKL and clinical scores.

\begin{tabular}{lrcrc}
\hline & $r$ & $p$ & $r$ & $p$ \\
& $($ Spearman $)$ & $($ Spearman $)$ & (Pearson) & (Pearson) \\
\hline PD duration & $\mathbf{0 . 4 0}$ & $\mathbf{0 . 0 0 3 8}$ & $\mathbf{0 . 3 1}$ & $\mathbf{0 . 0 2 4 1}$ \\
UPDRS III & -0.12 & 0.4397 & -0.19 & 0.2224 \\
FOG-Q & $\mathbf{0 . 4 1}$ & $\mathbf{0 . 0 0 2 4}$ & $\mathbf{0 . 4 0}$ & $\mathbf{0 . 0 0 2 9}$ \\
NMSS & $\mathbf{0 . 4 4}$ & $\mathbf{0 . 0 0 1 3}$ & $\mathbf{0 . 4 1}$ & $\mathbf{0 . 0 0 2 4}$ \\
RBDSQ & $\mathbf{0 . 5 1}$ & $\mathbf{0 . 0 0 0 2}$ & $\mathbf{0 . 5 2}$ & $\mathbf{0 . 0 0 0 1}$ \\
MMSE & -0.15 & 0.3325 & -0.18 & 0.2620 \\
ACE-R & 0.21 & 0.1769 & 0.24 & 0.1352 \\
BDI & -0.10 & 0.5351 & -0.12 & 0.4314 \\
\hline
\end{tabular}

\section{Discussion}

As it can be seen, $D_{\mathrm{KL}}$ is correlated significantly with PD duration which is an important finding, because then $D_{\mathrm{KL}}$ could be considered as a PDprogress monitoring feature. In fact, this finding supports the results of Rusz et al $l^{37}$ who reported that impaired vowel articulation may be even considered as a possible early marker of PD. Both correlations are positive, therefore it may be concluded that vowel articulation distortion is getting more significant as the disorder progresses. Another significant correlation was identified with the total score of FOG-Q. 
FOG and HD are both disabling axial symptoms of $\mathrm{PD}$, and they may point to an existence of possible common pathophysiological mechanisms, as it has already been reported $[38$ Moreover, Goberman 41 concluded that impaired phonation in PD patients is one of the speech disorders linked to gait difficulties. However, this author reported significant correlations with irregular pitch fluctuations as measured by standard deviation of fundamental frequency. In the present study, it was observed that vowel articulation distortion is linked to gait difficulties as well. The next significant correlation was identified with the total score of NMSS. To the best of our knowledge, there are no publications relating PD voice disorders and NMSS scores. NMSS is a very heterogeneous scale and its total score is given as a sum of 30 sub-scores. Therefore, it can only be hypothesized that the significant relation is present as besides all, the NMSS is assessing sleep disorders, for instance the REM sleep behavioral disorder. A significant correlation with this disorder is observed using the RBDSQ score. Speech/voice disorders are prodromal markers of $\mathrm{PD}$ in patients with the REM sleep behavioral disorder $\stackrel{42}{ }$ Specifically, Rusz et al $]^{43}$ found out that $88 \%$ of patients with this disorder had some kind of speech impairment. However, the present work is the first one confirming these associations based on vowel articulation disorder. Incidentally, significant correlations with the rest of clinical scores have not been identified in the present research. Other results are relatively less consistent. Different factors could explain inconsistent results, as the variability and low reliability of subjective scoring scales. No matter how well-designed the protocols may be or well-trained raters are involved, a human subjective factor is implicit and difficult to be removed. It must be said in this respect that subjectivity has to see with the proper conception and implementation of scores as UPDRS, FOG, NMSS, RBDSQ, MMSE, ACE-R or BDI, strongly dependent on patients' and/or clinicians' appreciations. Scoring specific functions on scales based on nonnumeric estimations or on behavioral features, not easily translatable to numeric scales, presents always a high degree of uncertainty and randomness, which is difficult to remove even using methods to evaluate raters for lack of subjectivity. At least three aspects of subjectivity may affect test scoring: ambiguity factors hidden in the test conception, subjective perception by raters, and unpredictable behavior of patients regarding medication and emotional factors. From the results in Table 2 it seems that PD duration as an objective concept, related to the time since PD was first diagnosed, is a reliable factor. Of course, this score is not free from uncertainty, as some patients may be diagnosed in a more advanced stage than others. UPDRS-III is oriented to describe general motor symptoms, not oriented specifically to speech, therefore it may not be quite precise in this respect. Freezing of Gait seems to be a quite well established concept, as well as the tests for NonMotor Symptoms, or sleeping disorders (RBDSQ), because they rely on scoring well observable facts. This does not seem to be the case of Mini-Mental State Examination, Addenbrooke's Cognitive evaluation or BDI, all of them based on assessing cognitive facts, by far more elusive than other observables. It may be assumed that neuromotor, behavioral nonneuromotor and sleeping facts are less affected by subjective perception by raters, and that the tests designed for their estimation are less ambiguous. The tests based on cognitive facts may be less free of ambiguity and self-subjectiveness. In any case, it is highly intriguing that the scores suffering from a worse correlation are those mainly based on cognitive factors. These facts stress the need of developing objective scoring methods even more. But in general, it may be concluded that a certain degree of correlation between formant dynamics and several motor and nonmotor scoring scales exist in PD, and could be conveniently exploited when fused with other articulation static features as VSA or FCR.

A very interesting question at the view of the results is the modification introduced in $D_{\mathrm{KL}}$ as expressed in (7). Taking the absolute value of the logarithm grants that nonoverlapping parts of the distributions are accounted always favoring distance. Besides, using the geometric mean of the model set pdf's, grants that distances are evaluated with reference to the absolute logarithmic ratio, especially meaningful in the areas dominated by the maxima of the model geometric mean. A specific reflection has to be devoted to the use of Mutual Information Measurements as $D_{\mathrm{KL}}$ instead of classical statistical approaches based on Parametric Descriptive Statistics (means, deviations, etc.). In doing so, it must be taken into account that Mutual Information Contents are based on using whole probability 
density functions to estimate similarities and divergences, instead of a couple of descriptive parameters as means and standard deviations, which have been estimated most of the times assuming that statistical distributions are Gaussian-like without any formal assessment. In this sense, an $N$-count normalized histogram is an $\mathrm{N}$-dimensional description of a process in the domain of sample values. Entropy and Mutual Entropy are good descriptors of statistical complexity, and Kullback-Leibler Divergence, or the more symmetrical Jensen-Shannon and similar measurements, may describe the similarity of statistical distributions quite well. $\underline{44 / 45}$

Another controversial experiment design decision was the selection method of the normative speaker subset used in estimating $D_{\mathrm{KL}}$. Its number (eight speakers only) may seem rather low to grant enough statistical dispersion. This decision was a deliberate option to bring to attention the capability of feature selection based on Information Theory criteria to represent variability. In dealing with separating features from pathological subjects with respect to normative subjects, it must be taken into account that normative features present much less dispersion than pathological ones. Therefore, normative clusters need not be of large size, it will suffice that their size be the right one to represent a wider set of subjects as far as variability is well represented. In the present case, the availability of a divergence estimation as $D_{\mathrm{KL}}$ allowed conforming unusually low normative datasets. The whole set of 53 normative speakers was rated, each one against the rest, and a minimum cluster was defined. Later on, each feature set from each subject was confronted with the cluster. If the $D_{\mathrm{KL}}$ between the subject and the cluster was under a given threshold, the subject was disregarded, as that dataset did not convey enough information. If the divergence was above the threshold, the new subject was added to the cluster, as this dataset conveyed relevant information. The information contents of the cluster as a function of the number of members were estimated. The representative members were selected to minimize size and maximize information contents.

A limitation of the study is the sample size and gender orientation of the PD database used in the study including only male subjects. It must be said in this respect, that producing a database including important clinical information, as the results of different motor, nonmotor and cognitive tests is not a simple task, and requires an important effort in time. Obtaining reliable records and test scores is a cumbersome task which has to be carried out by neurologists and clinical psycholinguists, who have to complete an exhaustive scoring for each patient. Other databases include meager clinical information, and are not suitable for this kind of studies. The results presented here are from a set of male PD patients, because at the time of publication the female dataset was not complete yet. Another factor, which complicates female speech studies is the fact that female voice is more sensitive to other factors as hormonal, 46 and need to be treated with other protocols to better differentiate presbyphonic, hormonal and neuromotor factors. For these reasons, results from the female database will be addressed in a separate paper in the near future.

An important open question is how well kinematic features may perform in differentiating and scoring PD by speech disorder correlates. The interested reader may check several recent publications on this respect, involving the use of pattern recognition methods as probabilistic neural networks, support vector machines, or classification trees, using different sets of biomarkers to assess disease detection and progression! $10,11|34| 36 \mid 47$

A final consideration has to see with the differential neuromotor control of tongue and associated articulation organs with respect to other muscles in the limbs. It is accepted that distal extremities are affected first along with disease progression, before the decline of neuromotor control affects the whole body. In this sense, the methodology presented in this research can be of most interest to investigate axial neuromotor functions, complementing other procedures more concentrated in gait or upper limb control, as handwriting.

\section{Conclusions}

The main conclusion which can be derived from the present study is that an explicit correlation may be observed among information divergence estimates and several clinical scores classically used in patient inspection and evaluation of PD. For instance, a statistically relevant relationship is established between the time interval since PD was first diagnosed (PD duration) and the $D_{\mathrm{KL}}$ as revealed in Table 2 Other 
relevant results are the positive correlations of $D_{\mathrm{KL}}$ to clinically meaningful indices as freezing of gait, nonmotor symptoms, and sleep disorders, all these in reference to the male subset. Especially relevant is the relation between FOG and HD, as both are axial symptoms sharing some pathophysiological mechanisms. Besides, a tight relation between sleep and speech disorders has been reported. Results from a similar study on female voices are still pending on the availability of more samples, and will be ready in the next future. Another important conclusion is that it becomes clear that probability distributions of articulation dynamics can be used to estimate distances on normative behavior in relating many patterns considered by clinicians as clear biomarkers to monitor PD progression in terms of Information Theory. This may open ways for establishing more accurate and objective inspection protocols including specific speech behavior correlates based on articulation gestures, as it is well known that speech is one of the earliest and more affected abilities impaired by $\mathrm{PD}$. Besides, speech is a very convenient reference, as it is ubiquitous, easy to record, and feasible for feature estimation using not very sophisticated or expensive resources. In this sense, new inspection protocols based on composite scores have to be designed to have speech into account in the characterization of neuromotor deterioration induced by this pathology.

\section{Acknowledgments}

This work is being funded by grants TEC201677791-C4-4-R from the Ministry of Economic Affairs and Competitiveness of Spain, TekaPark FGCSIC CENIE-0348_CIE_6_E (InterReg Programme) and 16-30805A, SIX Research Center (CZ.1.05/2.1.00/03.0072), and LOl401 from the Czech Republic Government.

\section{References}

1. C. D. Marsden, Parkinson's disease, J. Neurol. Neurosurg. Psychiatry 57 (1994) 672-681.

2. O. Darbin, X. Jin, C. von Wrangler, K. Schwabe, A. Nambu, D. K. Naritoku, J. K. Krauss and M. Alam, Relationship between entropy of spike timing and firing rate in entopeduncular nucleus neurons in anesthetized rats: Function of the nigrostriatal pathway, Int. J. Neural Syst. 26(2) (2016) 1550038 .
3. O. Hornykiewicz, Biochemical aspects of Parkinson's disease, Neurology 51(2) (1998) S2-S9.

4. J. Jankovic, Parkinson's disease: Clinical features and diagnosis, J. Neurol. Neurosurg. Psychiatry 79(4) (2008) 368-376.

5. P. Brodal, The Central Nervous System: Structure and Function, 3rd edn. (Oxford University Press, 2003).

6. R. Yuvaraj, M. Murugappan, U. Rajendra, H. Adeli, N. M. Ibrahim and E. Mesquita, Brain functional connectivity patterns for emotional state classification in Parkinson's disease patients without dementia, Behav. Brain Res. 298 (2016) 248-260.

7. W. Dauer and S. Przedborski, Parkinson's disease: Mechanisms and models, Neuron 39(6) (2003) 889909.

8. M. C. De Rijk, L. J. Launer, K. Berger, M. M. B. Breteler, J. F. Dartigues, M. Baldereschi, L. Fratiglioni, A. Lobo, J. Martínez, C. Trenkwalder and A. Hofman, Prevalence of Parkinson's disease in Europe: A collaborative study of population-based cohorts, Neurology 54 (2000) 21-23.

9. L. O. Ramig, C. Fox and S. Sapir, Speech treatment for Parkinson's disease, Expert Rev. Neurother. 8(2) (2008) 297-309.

10. L. Brabenec, J. Mekyska, Z. Galaz and I. Rektorova, Speech disorders in Parkinson's disease: Early diagnostics and effects of medication and brain stimulation, J. Neural Transm. 124(3) (2017) 303-334.

11. J. Mekyska, E. Janusova, P. Gómez, Z. Smekal, I. Rektorova, I. Eliasova, M. Kostalova, M. Mrackova, J. B. Alonso, M. Faúndez and K. López de Ipiña, Robust and complex approach of patohogical speech signal analysis, Neurocomputing 167 (2015) 94-111.

12. S. Sapir, L. O. Ramig, J. L. Spielman and C. Fox, Formant centralization ratio: A proposal for a new acoustic measure of dysarthric speech, J. Speech Lang. Hear. Res. 53 (2010) 114-125.

13. Movement Disorder Society Task Force on Rating Scales for Parkinson's Disease, The Unified Parkinson's Disease Rating Scale (UPDRS): Status and recommendations, Mov. Disord. 18 (2003) 738-750.

14. C. G. Goetz, W. Poewe, O. Rascol, C. Sampaio, G. T. Stebbins, D. Counsell, N. Giladi, R. G. Holloway, C. G. Moore, G. K. Wenning, M. D. Yahr and L. Seidl, Movement disorder society task force report on the Hoehn and Yahr staging scale: Status and recommendations the movement disorder society task force on rating scales for Parkinson's disease, Mov. Disord. 19 (2004) 1020-1028.

15. N. Giladi, H. Shabtai, E. S. Simon, S. Biran, J. Tal and S. D. Korczyn, Construction of freezing of gait questionnaire for patients with Parkinsonism, Park. Relat. Disord. 6(3) (2000) 165-170.

16. K. R. Chaudhuri, P. Odin, A. Antonini and P. Martínez, Parkinson's disease: The nonmotor issues, Park. Relat. Disord. 17(10) (2011) 717-723. 
17. K. Stiasny-Kolster, G. Mayer, S. Schafer, J. C. Muller, M. Heinzel-Gutenbrunner and W. H. Oertel, The rem sleep behavior disorder screening questionnaire-a new diagnostic instrument, Mov. Disord. 22(16) (2007) 2386-2393.

18. K. Stiasny-Kolster, F. Sixel-Döring, C. Trenkwalder, M. Heinzel-Gutenbrunner, K. Seppi, W. Poewe, B. Högl and B. Frauscher, Diagnostic value of the REM sleep behavior disorder screening questionnaire in Parkinson's disease, Sleep Med. 16 (2015) 186189.

19. A. F. Leentjens, F. R. Verhey, G. J. Luijckx and J. Troost, The validity of the Beck Depression Inventory as a screening and diagnostic instrument for depression in patients with Parkinson's disease, Mov. Disord. 15(6) (2000) 1221-1224.

20. D. Burdick, B. Cholerton, G. S. Watson, A. Siderowf, J. Q. Trojanowski, D. Weintraub, B. Ritz, S. L. Rhodes, R. Rausch, S. A. Factor, C. Wood, J. F. Quinn, K. A. Chung, S. Srivatsal, K. L. Edwards, T. J. Montiene, C. P. Zabetian and J. B. Leverenz, People with Parkinson disease and normal MMSE score have a broad range of cognitive performance, Mov. Disord. 29(10) (2014) 1258-1264.

21. E. Mioshi, K. Dawson, J. Mitchell, R. Arnold and J. R. Hodges, The Addenbrooke's cognitive examination revised (ACE- R): A brief cognitive test battery for dementia screening, Int. J. Ger. Psychiatry 21(11) (2006) 1078-1085.

22. P. Gómez, D. Palacios, A. Gómez, L. M. Ferrández, A. Álvarez, R. Martínez and V. Nieto, Relating facial myoelectric activity to speech formants, Proc. of IWINAC 2017, Lect. Notes Comp. Sci. 10338 (2017) 520-530.

23. J. R. Deller, J. G. Proakis and J. H. L. Hansen, Discrete-Time Processing of Speech Signals (Macmillan, New York, 1993).

24. J. Y. Lee, J. W. Kim, W. Y., J. M. Kim, T. B. Ahn, H. J. Kim, J. Cho and B. S. Jeon, Daily dose of dopaminergic medications in Parkinson's disease: Clinical correlates and a posteriori equation, Neurol. Asia 15(2) (2010) 137-143.

25. J. F. Demonet, G. Thierry and D. Cardebat, Renewal of the neurophysiology of language: Functional neuroimaging, Physiol. Rev. 85 (2005) 49-95.

26. A. G. Hannam, I. Stavness, J. E. Lloyd and S. Fels, A dynamic model of jaw and hyoid biomechanics during chewing, J. Biomech. 41 (2008) 1069-1076.

27. V. Sanguinetti, R. Laboissière and Y. Payan, A control model of human tongue movements in speech, Biol. Cybern. 77 (1997) 11-22.

28. P. Gómez, D. Palacios, A. Gómez, J. M. Ferrández, A. Álvarez, R. Martínez and V. Nieto, Relating facial myoelectric activity to speech formants, Springer Lect. Notes Comput. Sci. 10338 (2017) 520-530.
29. C. Dromey, G. O. Jang and K. Hollis, Assessing correlations between lingual movements and formants, Speech Commun. 55(2) (2013) 315-328.

30. C. T. Kelley, Iterative Methods for Optimization (SIAM, Philadelphia, 1999).

31. N. V. Norovkin, V. L. Chechurin and M. Hayakawa, Inverse Problems in Electric Circuits and Electromagnetics (Springer, 2007).

32. J. Blanchet, P. Glynn and S. Zheng, Empirical analysis of a stochastic approach for computing quasi-stationary distributions, EVOLVE-A Bridge between Probability Set Oriented Numerics, and Evolutionary Computation II (Springer, Berlin, 2013), pp. 19-37.

33. P. Gómez, D. Palacios, V. Rodellar, A. Álvarez, V. Nieto and R. Martínez, Parkinson's disease monitoring by biomechanical instability of phonation, $\mathrm{Neu}$ rocomputing 255 (2017) 3-16.

34. P. Gómez, J. Mekyska, J. M. Ferrández, D. Palacios, A. Gómez, V. Rodellar, Z. Galaz, Z. Smekal, I. Eliasova, M. Kostalova and I. Rektorova, Parkinson disease detection from speech articulation neuromechanics, Frontiers in Neuroinformatics, August 27 (2017); doi: 10.3389/fninf.2017.00056.

35. T. M. Cover and J. A. Thomas, Elements of Information Theory (Wiley, New York, 2006).

36. A. Tsanas, M. A. Little, P. E. McSharry, J. Spielman and L. O. Ramig, Novel speech signal processing algorithms for high-accuracy classification of Parkinson's disease, IEEE Trans. Biomed. Eng. 59 (2010) 1264-1271.

37. J. Rusz, R. Cmejla, T. Tykalova, H. Ruzickova, J. Klempir, V. Majerova, J. Picmausova, J. Roth and E. Ruzicka, Imprecise vowel articulation as a potential early marker of Parkinson's disease: Effect of speaking task, J. Acoust. Soc. Am. 134 (2013) 2171-2181.

38. N. Giladi, M. P. McDermott, S. Fahn, S. Przedborski, J. Jankovic, M. Stern, C. Tanner, Freezing of gait in PD: Prospective assessment in the DATATOP cohort, Neurology 56(12) (2001) 1712-1721.

39. S. Cantiniaux, M. Vaugoyeau, D. Robert, C. Horrelou-Pitek, J. Mancini, T. Witjas and J. P. Azulay, Comparative analysis of gait and speech in Parkinson's disease: Hypokinetic or dysrhythmic disorders? J. Neurol. Neurosurg. Psychiatry 81(2) (2010) 177-184.

40. L. Ricciardi, M. Ebreo, A. Graziosi, M. Barbuto, C. Sorbera, L. Morgante and F. Morgante, Speech and gait in Parkinson's disease: When rhythm matters, Park. Relat. Disord. 32 (2016) 42-47.

41. A. M. Goberman, Correlation between acoustic speech characteristics and non-speech motor performance in Parkinson's disease, Med. Sci. Monit. 11(3) (2005) 109-116. 
42. R. Postuma, A. E. Lang, J. F. Gagnon, A. Pelletier and J. Y. Montplaisir, How does parkinsonism start? Prodromal parkinsonism motor changes in idiopathic REM sleep behaviour disorder, Brain 35(Pt6) (2012) 1860-1870.

43. J. Rusz, J. Hlavnicka, T. Tykalova, J. Buskova, O. Ulmanova, E. Ruzicka and K. Sonka, Quantitative assessment of motor speech abnormalities in idiopathic rapid eye movement sleep behavior disorder, Sleep Med. 19 (2016) 141-147.

44. S. Pincus, Approximate entropy (ApEn) as a complexity measure, Chaos 5(1) (1995) 110-117.
45. M. Salicrú, D. Morales, M. L. Menéndez and L. Pardo, On the applications of divergence type measures in testing statistical hypotheses, J. Multivar. Anal. 51(2) (1994) 372-391.

46. J. Abitbol, P. Abitbol and B. Abitbol, Sex hormones and the female voice, J. Voice 13(3) (1999) 424-446.

47. T. J. Hirschauer, H. Adeli and J. A. Buford, Computer-aided diagnosis of Parkinson's disease using enhanced probabilistic neural network, J. Med. Syst. 39 (2015) 179. 